\title{
Basal cell carcinoma misdiagnosed as trichoepithelioma
}

Sung Jae Wee,

Myong Chul Park, Chan Min Chung

Department of Plastic and

Reconstructive Surgery, Hallym

University Sacred Heart Hospital,

Hallym University College of Medicine,

Anyang, Korea
Trichoepithelioma is a benign hair follicle tumor that can undergo malignant transformation into basal cell carcinoma in rare cases. Due to the similar clinical and histological features of trichoepithelioma and basal cell carcinoma, distinguishing between these types of tumors can be a diagnostic challenge. Punch biopsy obtains only a small sample of the entire lesion, and thus inherently involves a risk of misdiagnosis between histologically similar diseases. Therefore, if the possibility of misdiagnosis can reasonably be suspected, clinicians should conduct an excisional biopsy or immunohistochemical staining (e.g., CD10 and Bcl-2) to ensure an exact diagnosis. Although trichoepithelioma is benign, the surgical excision of solitary trichoepithelioma should be considered in order to avoid the possibility of malignant transformation, which has occasionally been documented for multiple familial trichoepitheliomas. Herein, we report a case that was initially misdiagnosed as trichoepithelioma before ultimately being diagnosed as basal cell carcinoma through excision and immunohistochemical staining.

Keywords: Carcinoma, basal cell / Diagnostic errors / Skin neoplasms

\section{INTRODUCTION}

Trichoepithelioma (TE) is a benign hair follicle tumor that can undergo malignant transformation into basal cell carcinoma (BCC) in rare cases [1]. Since TE and BCC share similar clinical and histological features, they are easily confused with one another, making the diagnosis difficult in some cases. Nonetheless, it is important to distinguish TE from BCC because the treatment modalities for these two conditions are completely different [2]. Therefore, misdiagnosis can lead to overtreatment or undertreatment [3]. For this reason, it is important for clinicians to choose a proper surgical treatment plan based on a thorough diagnosis by using a reliable diagnostic tool capable of distinguishing between these two tumor types with similar

\footnotetext{
Correspondence: Myong Chul Park

Department of Plastic and Reconstructive Surgery, Hallym University Sacred

Heart Hospital, Hallym University College of Medicine, 22 Gwanpyeong-ro

170beon-gil, Dongan-gu, Anyang 14068, Korea

E-mail: mpark@hallym.or.kr

Received April 24, 2020 / Revised May 23, 2020 / Accepted June 11, 2020
}

features. This paper reports a case of skin malignancy in the right medial canthal area that was initially misdiagnosed as benign TE through preoperative punch biopsy.

\section{CASE REPORT}

A 46-year-old woman with a skin-colored, round, protruding lesion on her right medial canthal area was referred to our outpatient clinic. She stated that she had received laser ablation treatment for the lesion at a local clinic 6 years ago, but the lesion did not shrink (Fig. 1). Its diameter was approximately $1.0 \times 0.5 \mathrm{~cm}$, and a punch biopsy performed by a dermatologist showed that it had no epidermal connection, no peripheral palisading, and concentric stroma (Fig. 2). Based on these findings, she was diagnosed with TE. Two years after the punch biopsy, she complained of discomfort due to recent growth of the mass and requested surgical removal.

A tangential excisional biopsy along the tumor margin was performed under local anesthesia. The excision site was har- 


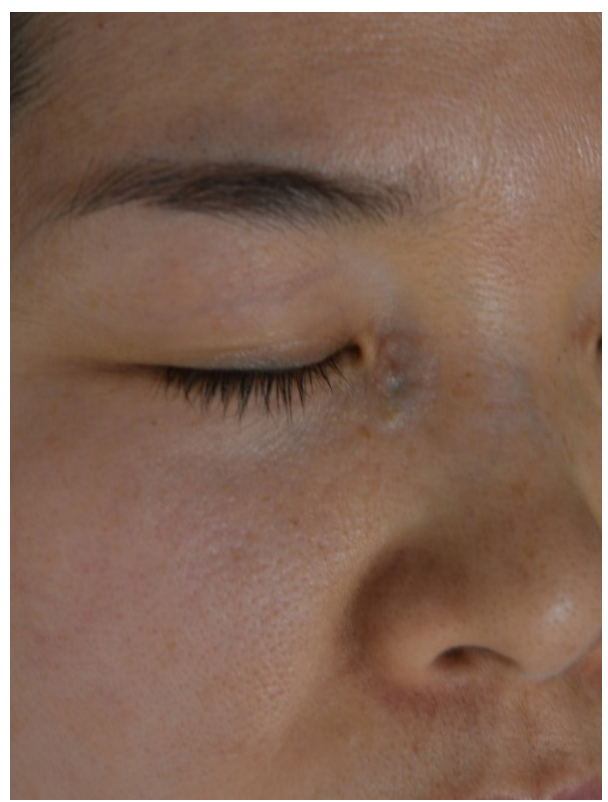

Fig. 1. Preoperative photograph of a 46-year-old woman with a $1.0 \times 0.5 \mathrm{~cm}$ mass on her right medial canthal area.

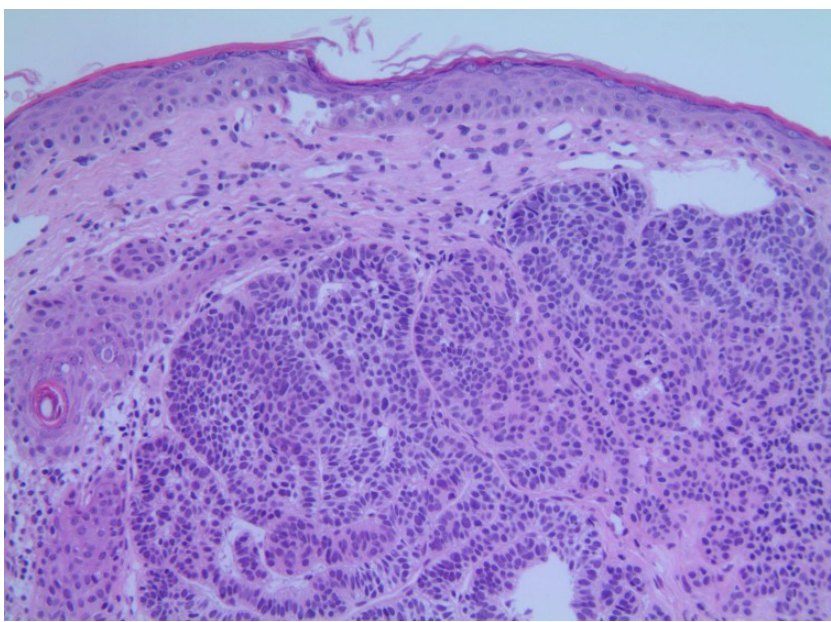

Fig. 2. Photomicrograph supporting the initial diagnosis as trichoepithelioma on punch biopsy. The findings included no epidermal connection, no peripheral palisading, and concentric stroma (H\&E, $\times 200)$.

vested with full-thickness skin from the right posterior auricular area and grafted with a tie-over dressing. The graft and donor sites had healed by 7 days postoperatively, but a histological examination confirmed that the mass was an atypical basaloid cell proliferative lesion with peripheral palisading (Fig. 3A). To evaluate the lesion more accurately, a pathological examination was performed using CD10 protein staining, with findings of partial positivity in the tumor cell nests from the excision specimen (Fig. 3B). The histopathological and immunohistochemical features of this tumor were characteristic of BCC, which was the final diagnosis.
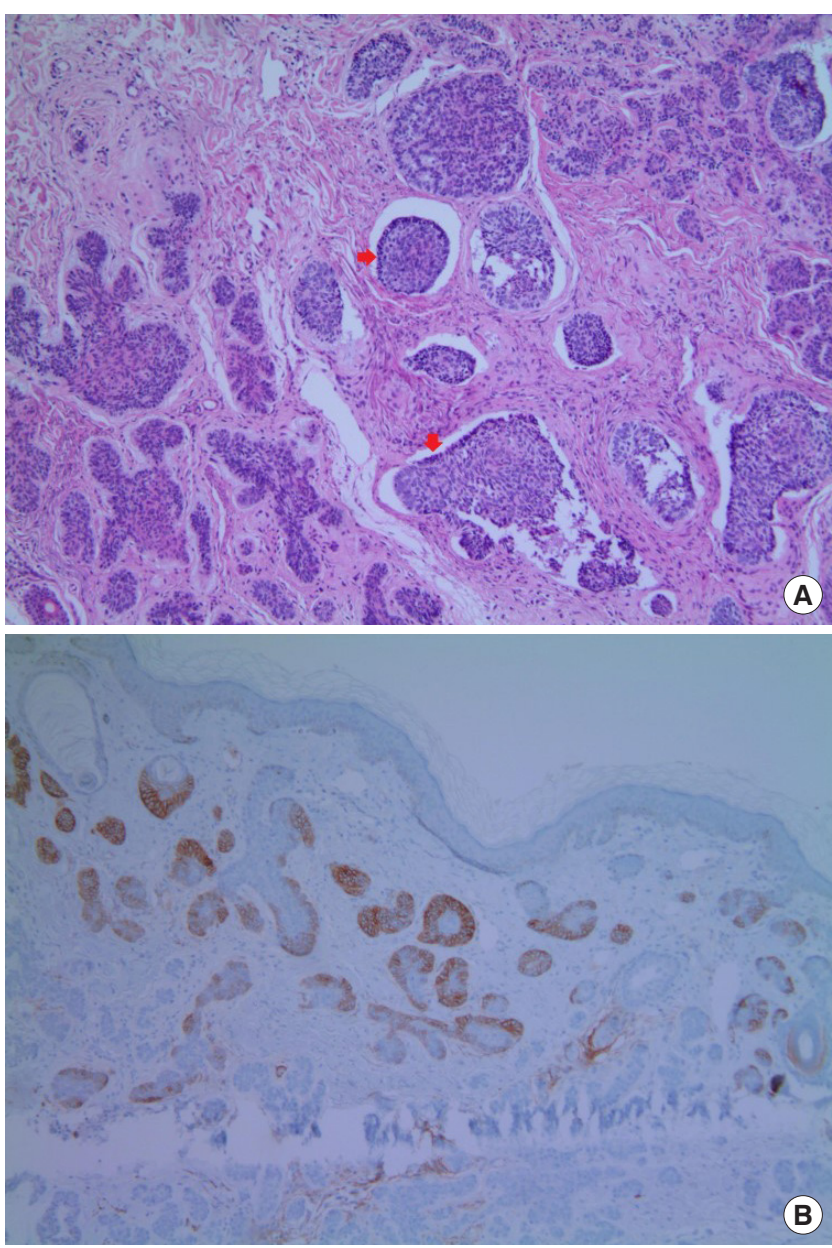

Fig. 3. Photomicrographs showing features of basal cell carcinoma. (A) Basaloid cell proliferation with peripheral palisades and a cleft between the tumor and stroma (arrows, H\&E, $\times 100$ ) and (B) positive results of CD10 protein staining in tumor cell nests $(\times 100)$.

An additional wide excision with a 4-mm safety margin was done with the patient under general anesthesia. After confirming the negative resection margin through an intraoperative frozen biopsy, another full-thickness skin graft was performed. The silk sutures in the skin were removed 7 days postoperatively, and the surgical site was observed to be completely epithelialized. A follow-up examination performed 1 month later showed no lingering wounds or complications (Fig. 4).

\section{DISCUSSION}

BCC, which is the most common form of primary skin cancer, primarily affects sun-exposed areas via exposure to ultraviolet radiation. It appears in the form of a well-defined nodule or plaque, and may be accompanied by ulceration or telangiectasia [4]. BCC is diagnosed through biopsy, and basaloid epithelial cell proliferation is a characteristic finding [5]. Since similar 


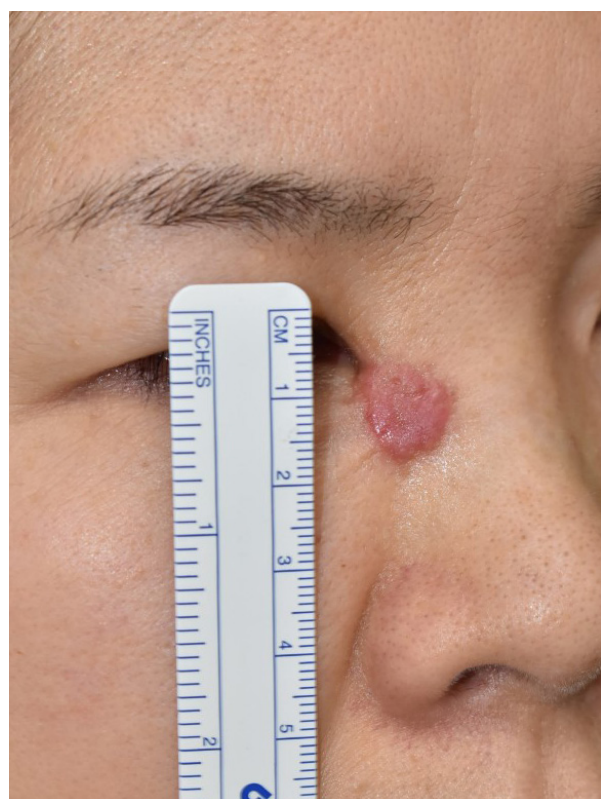

Fig. 4. Postoperative photograph 1 month after surgery.

findings are observed in cases of TE, which is a benign tumor, the possibility of an incorrect diagnosis should be kept in mind.

Histologically, BCC is characterized by tumor nests composed of basaloid cells with peripheral palisading. Epidermal connections of the tumor are relatively common, and stromal retraction is observed between the tumor and stroma [5]. In contrast, TE usually shows no epidermal connection, peripheral palisading is relatively inconspicuous, and stromal retraction is not clearly observed. Furthermore, the stroma is concentric and often accompanied by calcification [6]. However, it is challenging to distinguish these findings through a punch biopsy, from which only a small specimen is collected. Therefore, immunohistochemical staining is helpful for differentiating between these tumor types. Most notably, in TE, CD10 staining is positive in the stroma, but not in epithelial cells, whereas the opposite is true for BCC, as shown in our case (Fig. 3B) [6].

According to the National Comprehensive Cancer Network guideline, the standard treatment for $\mathrm{BCC}$ is wide excision with a 4-mm resection margin, and for high-risk BCC, Mohs micrographic surgery is performed to reduce the risk of recurrence [7]. In contrast, TE is a benign tumor, and simple excision is performed if necessary. Performing simple excision for BCC as a result of misdiagnosing it as TE constitutes undertreatment of the disease, increasing the risk of recurrence due to insufficient surgical excision. This issue may also have a meaningful impact on the patient's satisfaction and prognosis.

In this case, the patient was scheduled to undergo surgical excision for TE, but was subsequently lost to follow-up. Two years later, she visited our outpatient clinic because the mass was con- tinuing to grow, and it was eventually diagnosed as BCC based on the surgical excision specimen. There are two possible explanations for the discrepancy between these diagnoses.

First, it is possible that BCC was misdiagnosed as TE based on the initial punch biopsy. Since a punch biopsy only obtains a partial sample of the lesion, it is difficult to evaluate the entire lesion. Therefore, the diagnostic capability of punch biopsy for discriminating between two diseases with histologically similar patterns is limited. Misdiagnosis of the lesion is a reasonable possibility, since surgical excision was not performed, the patient was lost to follow-up, and immunohistochemical staining was not carried out at that time.

Another possibility is that TE developed into BCC. TE is a benign tumor of follicular origin that affects the face (e.g., the nasolabial fold area, nose, forehead, and upper lip) and occasionally undergoes malignant transformation [8]. Some reports have described the malignant transformation of TE into BCC, especially in cases of multiple TEs [9]. Pincus et al. [10] organized seven reports describing 11 patients, BCCs in individuals with multiple familial trichoepitheliomas (MFT), which showed the interval of about 36.1 years on average until there was the malignant transformation in MFT. However, the patient described herein had a small solitary TE, for which malignant transformation would be atypical. Therefore, our patient might be a rare case of malignant transformation from a solitary TE to BCC, which to our knowledge has rarely been reported previously [11].

In conclusion, TE and BCC are difficult to distinguish through visual inspection or punch biopsy. However, discriminating them is extremely important because the diagnosis affects the excision margin and has implications for patients' prognosis. In this case, it is unclear whether BCC was initially misdiagnosed as TE or TE transformed into BCC, but both possibilities furnish relevant lessons. First, clinicians should consider BCC in the differential diagnosis when punch biopsy reveals features of TE. As punch biopsy only obtains a small sample of the entire lesion, there is an inherent risk of misdiagnosing histologically similar diseases. Thus, if there is any reasonable likelihood of misdiagnosis, clinicians should conduct an excisional biopsy and/or immunohistochemical staining (e.g., CD10 and Bcl-2) to make a more accurate diagnosis. Second, although TE is benign and rarely undergoes malignant transformation, clinicians should consider surgical excision in cases of TE in light of the possibility that TE may develop into a malignancy. Hence, we report a rare case that was initially diagnosed as TE, but eventually diagnosed as BCC through excision and immunohistochemical staining. 


\section{NOTES}

\section{Conflict of interest}

No potential conflict of interest relevant to this article was reported.

\section{Ethical approval}

The study was approved by the Institutional Review Board of Hallym Sacred Heart Hospital (IRB No. 2019-12-005) and performed in accordance with the principles of the Declaration of Helsinki. Written informed consent was obtained.

\section{Patient consent}

The patient provided written informed consent for the publication and the use of her images.

\section{ORCID}

Sung Jae Wee https://orcid.org/0000-0002-2397-7450

Myong Chul Park https://orcid.org/0000-0002-0845-2672

Chan Min Chung https://orcid.org/0000-0002-2755-9440

\section{REFERENCES}

1. Mapar MA, Ranjbari N, Afshar N, Karimzadeh I, Karimzadeh A. Severely disfiguring multiple familial trichoepitheliomas with basal cell carcinoma. Indian J Dermatol Venereol Leprol 2014;80:349-52.

2. Kang KJ, Seo BF, Shin J, Kwon H, Byeon JH, Jung SN. Trichoepithelioma misdiagnosed as basal cell carcinoma. J Craniofac
Surg 2019;30:e197-9.

3. Stanoszek LM, Wang GY, Harms PW. Histologic mimics of basal cell carcinoma. Arch Pathol Lab Med 2017;141:1490-502.

4. Crowson AN. Basal cell carcinoma: biology, morphology and clinical implications. Mod Pathol 2006;19 Suppl 2:S127-47.

5. Sexton M, Jones DB, Maloney ME. Histologic pattern analysis of basal cell carcinoma: study of a series of 1039 consecutive neoplasms. J Am Acad Dermatol 1990;23(6 Pt 1):1118-26.

6. Calonje JE, Brenn T, Lazar AJ, Billings SD. McKee's pathology of the skin: with clinical correlations. 5th ed. Philadelphia: Elsevier; 2019.

7. Bichakjian CK, Olencki T, Aasi SZ, Alam M, Andersen JS, Berg D, et al. Basal cell skin cancer, version 1.2016, NCCN clinical practice guidelines in oncology. J Natl Compr Canc Netw 2016; 14:574-97.

8. Greywal T, Rubin AG, Jiang B. A rare presentation of basal cell carcinoma arising within trichoepithelioma: a diagnostic challenge. Cureus 2019;11:e5401.

9. Kallam AR, Satyanarayana MA, Aryasomayajula S, Krishna BA. Basal cell carcinoma developing from trichoepithelioma: review of three cases. J Clin Diagn Res 2016;10:PD17-9.

10. Pincus LB, McCalmont TH, Neuhaus IM, Kasper R, Oh DH. Basal cell carcinomas arising within multiple trichoepitheliomas. J Cutan Pathol 2008;35 Suppl 1:59-64.

11. Kim SH, Kim DW, Hwang JH, Kim KS, Lee SY. A rare development of basal cell carcinoma on trichoepithelioma in a chemical burn scar tissue: a case report. Medicine (Baltimore) 2018; 97:e12252. 